premotor/motor cortex and connections. A pontine 'coordination center' for laughter is postulated. Fibers from the periaqueductal grey, transmitting the signal to laugh, are located dorsally/tegmentally, whereas fibers inhibiting facial emotional expressions run ventrally from frontal motor areas, and may also involve the cerebellum. Two partially independent neuronal pathways are involved in the expression of laughter: 1) an 'involuntary' or 'emotionally driven' system, involving amygdala, thalamus, hypothalamus, subthalamus, and dorsal/tegmental brainstem; and 2) a 'voluntary' system originating in the premotor/frontal opercular areas, and passing via the motor cortex and pyramidal tract to the ventral brainstem. (Wild B, Rodden FA, Grodd W, Ruch W. Neural correlates of laughter and humour. Brain October 2003;126:2121-2138). (Respond: Dr Barbara Wild, Psychiatrische Universitatsklinik, Osianderstrasse 24, 72076 Tubingen, Germany).

COMMENT. In pediatric neurology practice, pathological laughter occurs in patients with Angelman syndrome, pseudobulbar palsy as in cerebral palsy and infantile hydrocephalus, hypothalamic hamartoma and gelastic epilepsy, infantile spasms (Lacy JR, Penry JK, 1976), and complex partial seizures. Gelastic seizures were controlled by removal of a cavernous hemangioma in the anterior cingulate gyrus in one child treated at Johns Hopkins Hospital, Baltimore, MD (Arroyo S et al. Brain 1993;116:757-780). Mirth and laughter were invoked by cortical stimulation of the parahippocampal and fusiform gyri in 2 additional children treated for complex partial seizures. (see Progress in Pediatric Neurology II, PNB Publ, 1994;pp41-42).

\title{
EVOLUTION OF GELASTIC EPILEPSY WITH HYPOTHALAMIC HAMARTOMA
}

The patterns of clinical presentation, evolution of the epilepsy, and electoclinical diagnostic features of hypothalamic hamartoma $(\mathrm{HH})$ in 19 patients $(8$ children and 11 adults), seen between 1991 and 2001, were evaluated at Kings College Hospital and the Institute of Epileptology, London, UK. Of 16 with early-onset epilepsy, 15 had gelastic seizures, and epilepsy began between 0 and 5 years (mean, 2.1 years) in 14, one having infantile spasms in infancy. Precocious puberty (PP) developed in 6, and learning disability in 12. MRI was diagnostic in all. In 8 older patients in the early-onset group, $\mathrm{HH}$ was diagnosed between 19 and 54 years (mean, 33 years). Gelastic seizures disappeared in 2 , or were reduced to "an urge to laugh" in adolescence and young adulthood. They were replaced by multiple seizure types and evolved into mainly complex partial epilepsy ( 5 of 8 adults), tonic seizures, or symptomatic generalized epilepsy with atypical absences, drop attacks, and secondarily generalized seizures with cognitive impairment ( 3 of 8 adults). Of 3 adult-onset patients, only one had gelastic seizures manifested by inappropriate smiling but no laughing, epilepsy was mild, and cognition normal. Of 14 patients with intrahypothalamic hamartomas, all had moderate to severe gelastic epilepsy, 5 had PP, and 13 had early onset epilepsy, whereas of 5 with parahypothalamic hamartomas, only 2 had gelastic seizures, and 3 had late-onset epilepsy. The larger the $\mathrm{HH}$, the greater the number of seizure types associated. Minimally invasive stereotactic thermocoagulation appeared to be superior to open surgical resection of the HH in less severe cases. (Mullatti N, Selway 
R, Nashef L, et al. The clinical spectrum of epilepsy in children and adults with hypothalamic hamartoma. Epilepsia October 2003;44:1310-1319). (Reprints: Dr N Mullatti, Department of Clinical Neurophysiology, Kings College Hospital, Denmark Hill, Lonson SE5 9RS, UK).

COMMENT. Gelastic seizures are the rule with hypothalamic hamartoma $(\mathrm{HH})$ and early-onset epilepsy, but they frequently evolve into other and milder types of epilepsy in adolescence and adulthood. In adult-onset epilepsy with $\mathrm{HH}$, gelastic seizures are less frequent and milder, and epilepsy is often of the complex partial, tonic, or atypical absence types. Cognitive and behavioral disorders are less frequent in adult-onset cases of epilepsy with $\mathrm{HH}$.

\section{SEIZURE DISORDERS}

\section{HIPPOCAMPAL ABNORMALITIES AFTER PROLONGED FEBRILE CONVULSIONS}

Hippocampal volume and T2 relaxation times were determined in an MRI study of 14 children with prolonged febrile convulsions (PFC) who were investigated, 1) within 5 days of a PFC, and 2) at follow-up 4-8 months after the acute study, at the Institute of Child Health, University College, and Great Ormond Street Hospital, London, UK. The initial study previously published (Scott et al, 2002) showed that children examined by MRI within $48 \mathrm{~h}$ of a PFC had large hippocampal volumes and prolongation of T2 relaxation times, whereas those with MRI delayed $>48 \mathrm{~h}$ but within 5 days of PFC had large hippocampal volumes and normal $\mathrm{T} 2$ relaxation time. The data were suggestive of hippocampal edema that is resolving within 5 days of a PFC.

Of 14 patients examined at 4-8 month follow-up, 4 had suffered further seizures: 2 had short FC, 1 had PFC and 1 had non-febrile seizures. Repeat MRI showed a significant reduction in hippocampal volume and $\mathrm{T} 2$ relaxation time compared to the first exam, and volumes were not different from a control population. In addition, hippocampal volume asymmetry was significantly increased at follow-up compared to initial data, findings consistent with injury and neuronal loss associated with PC. It is postulated that some selectively vulnerable patients may develop mesial temporal sclerosis after a lag period of several years following the PFC or alternatively, the hippocampal asymmetry may represent a post-acute edema return to a pre-existing hippocampal abnormality that antedates the PFC. (Scott RC, King MD, Gadian DG, Neville BGR, Connelly A. Hippocampal abnormalities after prolonged febrile convulsion: a longitudinal MRI study. Brain November 2003;126:2551-2557). (Respond: Dr Rod C Scott, The Wolfson Centre, Mecklenburgh Square, London WC1N 2AP, UK).

COMMENT. In patients with intractable complex partial seizures, those with mesial temporal sclerosis (MTS) had a history of PFC in $33 \%$ whereas those without MTS had PFC in only 7\% (Falconer, MA, 1972, 1976; see Nelson and Ellenberg, 1981). A causative association between prolonged or complex FC and hippocampal injury and MTS is suggested by clinical and animal studies, but a pre-existing subtle hippocampal maldevelopment is possible in some cases of complex partial epilepsy. 